\title{
Two methods for transmission line simulation model creation based on time domain measurements
}

\author{
D. Rinas and S. Frei \\ Dortmund University of Technology, Dortmund, Germany
}

\begin{abstract}
The emission from transmission lines plays an important role in the electromagnetic compatibility of automotive electronic systems. In a frequency range below $200 \mathrm{MHz}$ radiation from cables is often the dominant emission factor. In higher frequency ranges radiation from PCBs and their housing becomes more relevant. Main sources for this emission are the conducting traces. The established field measurement methods according CISPR 25 for evaluation of emissions suffer from the need to use large anechoic chambers. Furthermore measurement data can not be used for simulation model creation in order to compute the overall fields radiated from a car. In this paper a method to determine the far-fields and a simulation model of radiating transmission lines, esp. cable bundles and conducting traces on planar structures, is proposed. The method measures the electromagnetic near-field above the test object. Measurements are done in time domain in order to get phase information and to reduce measurement time. On the basis of near-field data equivalent source identification can be done. Considering correlations between sources along each conductive structure in model creation process, the model accuracy increases and computational costs can be reduced.
\end{abstract}

\section{Introduction}

Electromagnetic Compatibility plays an important role in the development of automotive systems. Beside the radiating cable bundles integrated Electronic Control Units (ECUs) are sources for electromagnetic emissions.

Standardized component field measurement methods, like the ALSE antenna method provided in CISPR 25 for evaluation of electro-magnetic emissions from automotive sys-

Correspondence to: D. Rinas

(denis.rinas@tu-dortmund.de) tems, suffer from the need of large and expensive anechoic chambers. Also a single field strength value is often not sufficient to characterize the EMI behavior of a complex system. Furthermore it is not possible to use the measurement data for behavioral simulation model creation. Having simulation models, a statement about the radiating electromagnetic fields can already be made in early phases of development.

Basically the electro-magnetic emission must be distinguished in the emission of circuit boards and their housing and the emission of their connecting cable bundles. Where in lower frequency range up to $200 \mathrm{MHz}$ radiation from the bundles is dominant the importance of emission from ECUs grows with the rising frequency.

In Rinas et al. (2010) a method for estimating the emissions from cable bundles is presented. Here the electromagnetic near-field at several points near a cable bundle is measured. With measured data a single transmission line model with equivalent current distribution is generated. The measurements are done in Time Domain with a standard oscilloscope to get phase information and to reduce measurement time.

To create a behavioral model of PCB the electromagnetic near-field in a plane above the radiating structure is measured. Knowing the fields in an indefinitely extended plane above the test object all information is available to calculate any field vector above this plane (Balanis et al., 1996). This can be used to solve an inverse problem and to identify the equivalent sources of the planar structure. This approach is discussed in Vives-Gilabert (2007); Baudry et al. (2008); Isernia et al. (1996); and Tong et al. (2010). Several methods dealing with the inverse problem are ill-posed due to the presence of errors in measurement data, or suffer from the problem of converging to local minima. When optimization methods are applied this leads to long computation time (Isernia et al., 1996; Pierri et al., 1999). 


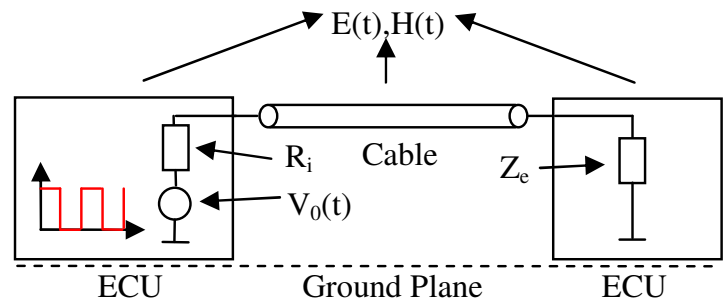

Fig. 1. Simple configuration to investigate.

As main sources for emission of PCBs are the conducting traces, the distribution of the approximating sources can be bounded to the routing geometry. Furthermore techniques for correlating the properties of the approximating sources to compute a more physically model can be integrated in the model creation process. This can reduce the number of free source parameters and computation time. Furthermore the possibility of error correction is given and accuracy of the results increases.

In this paper a method for source identification of transmission lines, in form of cables bundles and conducting paths on PCBs, with considering correlations between the sources is presented. Investigations are done in a frequency range up to $500 \mathrm{MHz}$. The generated model enables different types of postprocessing e.g. far field estimations.

\section{Source identification of cable bundles}

As shown in Rinas et al., 2010 the current distribution of a radiating single cable (Fig. 1) can be estimated by measuring the magnetic field in at least two field points along the cable.

When calculation is based on two measurement sets

$I_{z}=\frac{1}{2 Z}\left(V_{e}+Z I_{e}\right) e^{j \beta(l-z)}+\frac{1}{2 Z}\left(V_{e}-Z I_{e}\right) e^{-j \beta(l-z)}$

with

$$
\left(\begin{array}{l}
V_{e} \\
I_{e}
\end{array}\right)=\left[\begin{array}{ll}
a_{1} & b_{1} \\
a_{2} & b_{2}
\end{array}\right]^{-1} \cdot\left(\begin{array}{l}
I_{1}\left(z_{1}\right) \\
I_{2}\left(z_{2}\right)
\end{array}\right)
$$

leads to current $I_{z}$ at position $z$. Where $V_{e}$ and $I_{e}$ are voltage and current at the end of transmission line, $Z$ is the wave impedance, $\beta$ the propagation constant, $l$ the length of the line, $a$ and $b$ include the propagation functions and $I_{1}$ and $I_{2}$ are the current values corresponding to the magnetic field data.

The current distribution can also be estimated directly by increasing the amount of measured field points along the cable depending on the frequency with respect to

$N=l / \Delta d$

$\Delta d>\frac{1}{10} \lambda$

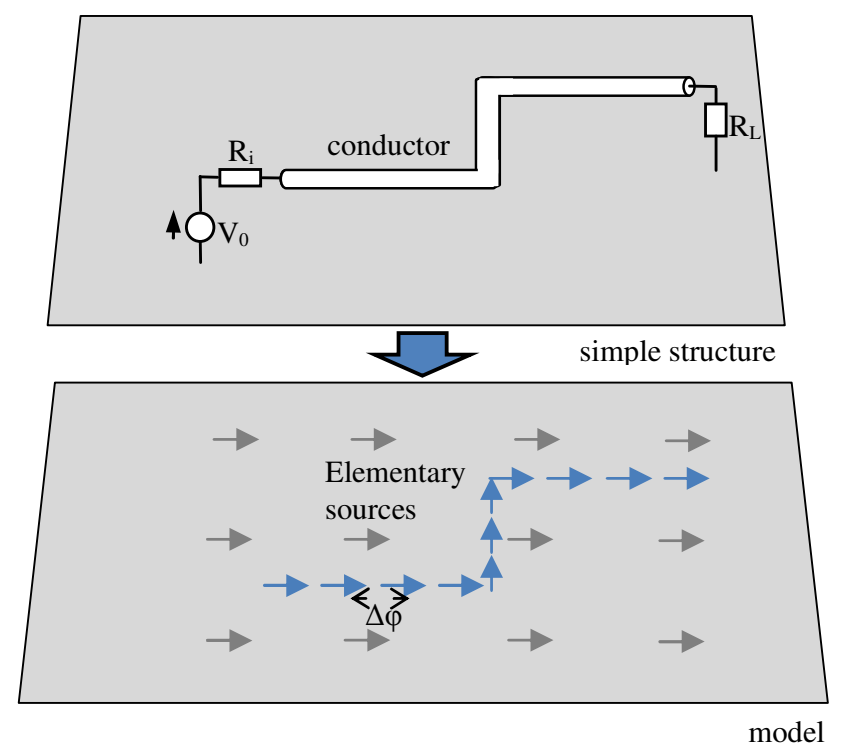

Fig. 2. Drawin g of the test board (above), model of the board (below) with equally distributed sources (grey) and sources only along the current path (blue).

where $N$ is the amount of measurement points and $\Delta d$ the spatial discretization width between the neighboring points. For achieving $I_{z}$ the discrete current values are approximated to a sinusoidal function with nonlinear regression algorithms.

In order to create an equivalent emission model elementary electric dipoles are placed in a line along the cable path in a height $h$ over ground adopted from the cable, following 3 . The dipole moments $M_{1} \ldots M_{K}$ are calculated with

$M_{k}=\Delta d \frac{I_{k}+I_{k+1}}{2}$

where $I_{k}$ is the discretized current $I_{z}$.

\section{Source identification of conducting traces on planar structures}

According to Table 2 transmission lines on planar structures, esp. PCBs, can also be approximated with elementary dipoles. With knowledge of the current paths, e.g. based on CAD-data, the approximating dipoles can be distributed along these paths (Fig. 2). Where $R_{i}$ and $R_{L}$ are the terminating impedances and $V_{0}$ is the exciting input voltage. The number of dipoles is chosen following Eq. (3) This approach, in comparison with the method of equally distributed dipoles, can considerable reduce the amount of necessary elementary sources, decrease computational cost and increase the model accuracy. 


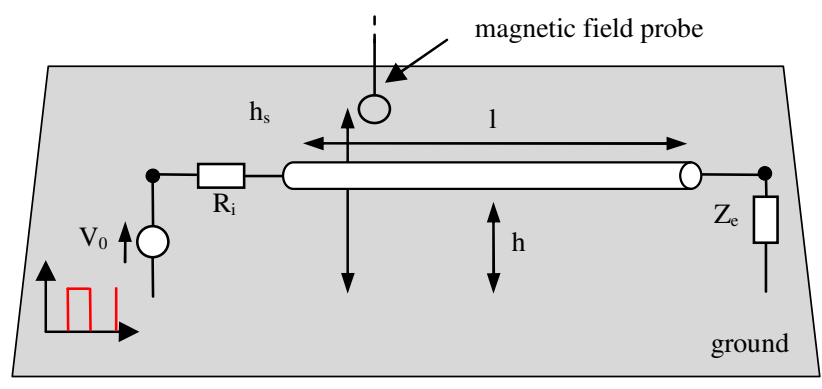

Fig. 3. Single conductor under test.

Dependencies between the neighboring elementary sources of each current path are used to correlate phases of the dipoles. For the spatial distribution $\Delta d$ the phase shift between two adjacent dipoles is set to

$\Delta \varphi=\frac{2 \pi}{\lambda} \Delta d$

as shown in Fig. 2. With adherence to Eq. (6) phase jumps between two sources different from $\Delta \phi$ are prevented and the model becomes more physically.

To compute the dipole moments $M$ the magnetic near-field has to be measured in a plane above the planar structure. Afterwards the system of equations

$I_{0}=\mathrm{A}^{-1} H$

where A contains the wave vector $k_{0}$ and the fixed geometric parameters for each dipole and $H$ the complex magnetic field at $K$ near-field points is solved.

For integrating the correlation between sources into model creation the inverse problem can be treated with the expandable minimization function $F$ following 4 .

$$
\begin{aligned}
& F=\left(\sum_{m=1}^{M} \alpha_{m}\left(x_{1}, y_{1}, z_{1}\right) M_{m} e^{-j \varphi_{m}}, \ldots,\right. \\
& \left.\sum_{m=1}^{M} \alpha_{m}\left(x_{N}, y_{N}, z_{N}\right) M_{m} e^{-j \varphi_{m}}\right)^{T} \\
& -\left(H_{0}\left(x_{1}, y_{1}, z_{1}\right), \ldots, H_{0}\left(x_{N}, y_{N}, z_{N}\right)\right)^{T} \rightarrow \text { Min }
\end{aligned}
$$

With $\alpha_{m}$ are the fixed geometric parameters at observation point $x_{n}, y_{n}, z_{n}$, the amplitude and $M_{m}$ is and the phase $e^{-j \phi m}$ of each dipole. $H_{0}\left(x_{n}, y_{n}, z_{n}\right)$ stands for the measured reference field at observation points. Optimization methods are used for solving the minimization problem.

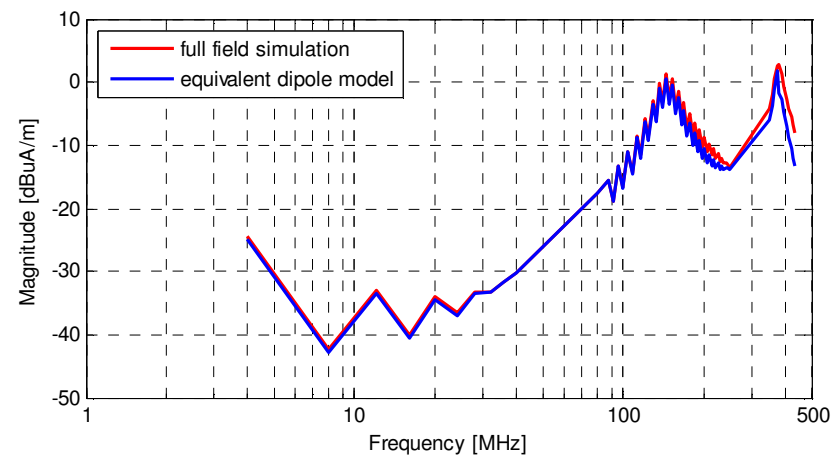

Fig. 4. Magnetic field at $p_{1}$ in comparison with full field simulation.

\begin{tabular}{|c|c|c|}
\hline \multirow{2}{*}{ Frequency } & \multicolumn{2}{|c|}{ Magnitude $[\mathrm{dB} \mu \mathrm{A} / \mathrm{m}]$} \\
\hline & Sim. & Model \\
\hline $4 \mathrm{MHz}$ & 29.4963 & 26.3542 \\
\hline $20 \mathrm{MHz}$ & 32.4243 & 32.9482 \\
\hline $60 \mathrm{MHz}$ & 36.7945 & 37.3562 \\
\hline $124 \mathrm{MHz}$ & 43.1243 & 45.2324 \\
\hline $164 \mathrm{MHz}$ & 47.7698 & 50.1270 \\
\hline
\end{tabular}

Table 1. Magnetic Field of Measurement based Model at $p_{1}$ in comparsion with full field simulation.

\section{Results}

\subsection{Cable bundles}

This chapter presents the results for model creation process of a single radiating cable and a small bundle. Some results are presently derived from an electromagnetic full field solver (EMCoS Consulting and Software, www.emcos.com).

The input signal $V_{0}$ (Figs. 1 and 3 ) is a pulsed signal with an amplitude $V_{0}=5 \mathrm{~V}$, a fundamental frequency of $f_{0}=4 \mathrm{MHz}$, a pulse/pause ratio $t_{h} / t_{l}=1$, and a rising and falling edge of $t_{r, f}=2.5 \mathrm{~ns}$. Source impedance $R_{i}$ is $50 \mathrm{Ohm}$.

\subsubsection{Single conductor - measurement based results}

The cable (Fig. 3) consists of a single conductor placed in the height $\mathrm{h}=50 \mathrm{~mm}$ over a ground plane. It has a length of $1=490 \mathrm{~mm}$ and a thickness of $\mathrm{d}=1 \mathrm{~mm}$. It is terminated with a $Z_{e}=50 \Omega$ impedance. The magnetic field is measured at two positions $y_{1}=120 \mathrm{~mm}$ and $y_{2}=360 \mathrm{~mm}$ along the cable at a height $h_{S}=20 \mathrm{~mm}$ over ground.

With the identified sources the magnetic field is calculated at a position $p_{1}=(3,3,3)^{T}[\mathrm{~m}]$. Exemplary the fields of the fundamental wave and the 2th, 7th, 15th and 20th harmonics of the pulsed input signal are presented in Table 1. As a comparison a full field simulation of the cable under test given by a MoM solver (EMCoS Consulting and Software, www.emcos.com) is shown. The results agree with a maximum error of $3 \mathrm{~dB}$. 
Table 2. Near-Fields of different Models in Comparison with full field simulation.

\begin{tabular}{|c|c|c|}
\hline $\begin{array}{l}\text { Full field } \\
\text { simulation }\end{array}$ & 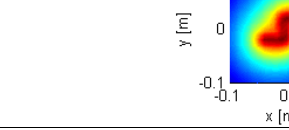 & 0.1 \\
\hline & Equally distributed sources & Sources along current path \\
\hline $\begin{array}{l}\text { No phase } \\
\text { correlation } \\
\text { of sources }\end{array}$ & 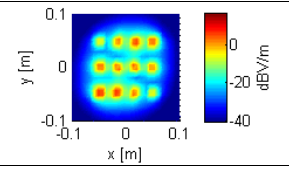 & 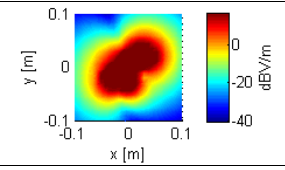 \\
\hline $\begin{array}{l}\text { With phase } \\
\text { correlation } \\
\text { of sources }\end{array}$ & 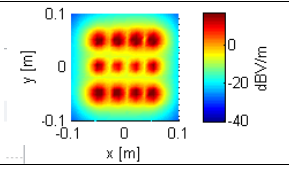 & 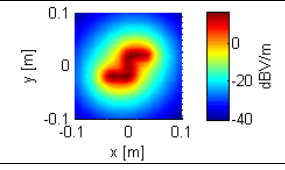 \\
\hline
\end{tabular}

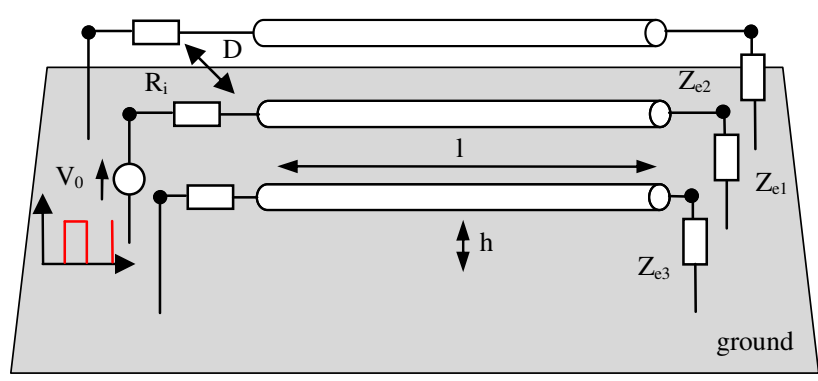

Fig. 5. Multiconductor under test.

\subsubsection{Single conductor - simulation based results}

The following investigations are presently based on computer simulations.

The cable (Fig. 3) consists of a single conductor placed in the height $\mathrm{h}=10 \mathrm{~mm}$ over the ground plane. It has a length of $1=600 \mathrm{~mm}$ and a thickness of $\mathrm{d}=0.3 \mathrm{~mm}$. It is terminated with a serial circuit of resistor $R_{e}=50 \Omega$ and inductance $L_{e}=$ $1 \mu \mathrm{H}$. The model parameters are taken from the cable under test. The source internal resistance is set to $R_{i}=50 \Omega$. The magnetic field is measured at two positions $y_{1}=200 \mathrm{~mm}$ and $y_{2}=400 \mathrm{~mm}$ along the cable at a height $h_{S}=20 \mathrm{~mm}$ over ground.

Figure 4 shows the magnetic field at point $p_{1}$ in a frequency range of $1 \mathrm{MHz}$ to $500 \mathrm{MHz}$. The results in comparison with the full field simulation agree with maximum error of about $3 \mathrm{~dB}$.

\subsubsection{Multiconductor - simulation based results}

The following investigations are presently based on computer simulations.

The multiconductor (Fig. 5) consists of three single conductors placed in the height of $\mathrm{h}=10 \mathrm{~mm}$ over ground

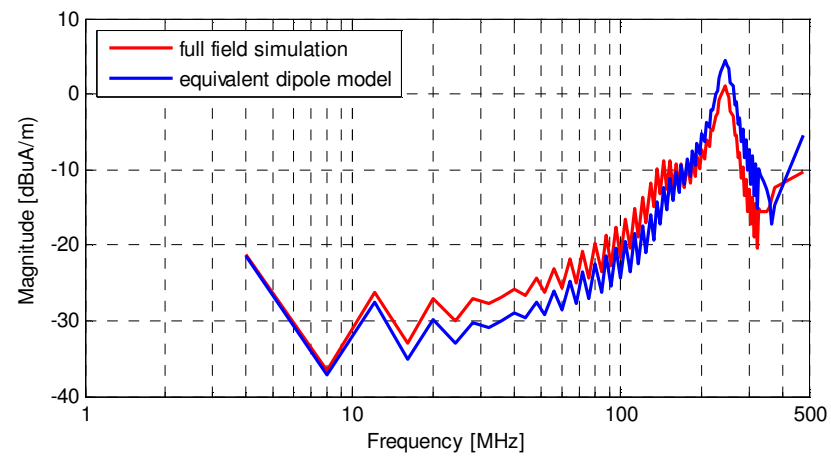

Fig. 6. Magnetic field at $p_{1}$ in comparison with full field simulation.

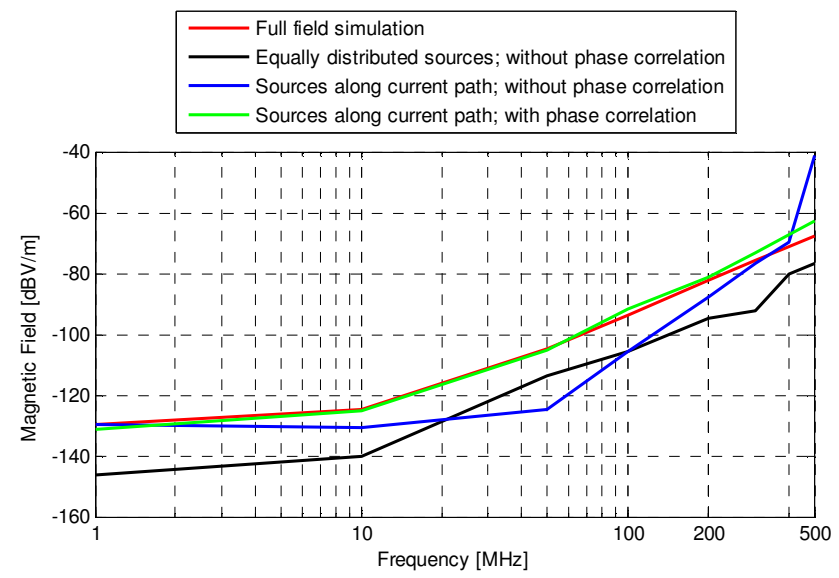

Fig. 7. Magnetic field at $p_{1}$ for different source distribution in comparison with full field simulation.

plane. The length of each conductor is $1=600 \mathrm{~mm}$; the thickness is $d=0.3 \mathrm{~mm}$ and they are arranged in a distance of $\mathrm{D}=5 \mathrm{~mm}$. The internal source resistances are set to $R_{i}=50 \Omega$. The terminations are $Z_{e 1}=50 \Omega \| j \omega \cdot 0.1 \mathrm{nF}$, $Z_{e 2}=50 \Omega, Z_{e 3}=50 \Omega+j \omega \cdot 1 \mu \mathrm{H}$. The excitation is impressed in the center conductor.

In Fig. 6 the magnetic field at point $p_{1}$ in comparison with the full field simulation is shown. The magnetic field agrees with a maximum error of $3 \mathrm{~dB}$ at most frequencies.

\subsection{Printed circuit boards - simulation based results}

A simple planar structure is analyzed. It consists of a $0.1 \times 0.1 \mathrm{~m}$ plane and a conductor with total length of $0.16 \mathrm{~m}$. The source voltage is 1 Volt; termination is a $50 \mathrm{Ohm}$ resistance. The magnetic field is measured in a height of $0.01 \mathrm{~m}$ over ground at 256 near-field points. The source parameters are computed with amplitude-data only. Simulated Annealing is used as optimization method for model parameter calculation.

In Table 2 near-field calculations from different models are shown and compared with a full field simulation (MoM) of 
the planar structure at a frequency of $100 \mathrm{MHz}$. Figure 7 shows the magnetic field at point $p_{1}=(3,3,3)^{T}[\mathrm{~m}]$ in comparison with the full field simulation in a frequency range of $1 \mathrm{MHz}$ to $500 \mathrm{MHz}$.

As presented, the field approximation shows better accuracy if the distribution of sources is matched with the current distribution depending on the conductor path. The result plots show also a smaller deviation if the phase correlation between sources is integrated in the model computation.

\section{Conclusions}

In this paper methods for source identification of radiating transmission lines are presented. The focus is on cables bundles and conducting traces on planar structures. Model accuracy is can be increased by considering correlation between the neighboring approximating sources.

The approaches were tested by simulations and first measurements and were compared with numerical full field simulation data.

\section{References}

CISPR 25 Ed.3: Vehicles, boats and internal combustion engines Radio disturbance characteristics - Limits and methods of measurement for the protection of on-board receivers, 2008.

Rinas, D., Niedzwiedz, S., and Frei, S.: Far Field Estimations and Simulation Model Creation from Cable Bundle Scans, EMC Europe, Wroclaw, 203-208, 2010.

Balanis, C. A.: Antenna Theory Analysis \& Design, Wiley, 1996.

Vives-Gilabert, Y.: Modélisation des emissions rayonées de composants électroniques, Université de Rouen, 2007.

Baudry, D., Kadi, M., Riah, Z., Arcambal, C., Vives-Gilabert, Y., Louis, A., and Mazari, B.: Plane wave spectrum theory applied to near-field measurements for electromagnetic compatibility investigations", IET Science, Measurement and Technology, 15 June 2008.

Isernia, T., Giovanni Leone, G. and Pierri, R.: Radiation Pattern Evaluation from Near-Field Intensities on Planes, IEEE Transaction on Antennas and Propagation, 44(5), 701-710, 1996.

Tong, X., Thomas, D. W. P., Nothofer, A., Sewell, P., and Christopoulos, C.: A Genetic Algorithm Based Method for Modeling Equivalent Emission Sources of Printed Circuits from Near-Field Measurements, APEMC Beijing, 2010.

Isernia, T., Leone, G., and Pierri, R.: Radiation pattern evaluation from near-field intensities on planes, IEEE Trans. Antennas Propogat., 44, 701-710, 1996.

Pierri, R., D'Elia, G., and Soldovieri, F.: A two probes scanning phaseless near-field far-field transformation technique, IEEE Trans. Antennas Propogat., 47, 792-802, 1999.

EMCoS Consulting and Software, www.emcos.com, January 2011. 